\title{
Factores determinantes de la ansiedad en atletas veteranos españoles*
}

\section{Determinants of Anxiety in Spanish Master Athletes}

Recibido: mayo 24 de 2013 | Revisado: octubre 17 de 2013 | Aceptado: octubre 17 de 2013

\author{
ANTONIO ZARAUZ SANCHO ** \\ Instituto de Educación Secundaria Azcona, Almería, España \\ FRANCISCO RUIZ-JUAN *** \\ Universidad de Murcia, España
}

doi:10.11144/Javeriana.UPSY13-3.fdaa

Para citar este artículo: Zarauz, A. \& Ruiz-Juan, F. (2014). Factores determinantes de la ansiedad en atletas veteranos españoles: Universitas Psychologica, 13(3), 1047-1058. http://dx.doi.org/10.11144/ Javeriana.UPSY13-3.fdaa

* Agradecimientos: este trabajo ha sido posible gracias a la inestimable colaboración prestada por D. Fernando Marquina Alonso, Responsable del Departamento de Atletas Veteranos de la Real Federación Española de Atletismo, y por D. Vicente Sánchez Blanco, webmaster de www.elatleta.com.

** Jefe Dpto. Educación Física y Deporte. I.E.S. Azcona. C/Santa Laura, 30. 04008 - Almería (Almería). Correo electrónico: tonizarauz@msn.com

**** Facultad de Ciencias del Deporte. C/ Argentina, s/n. 30720 - Santiago de la Ribera (Murcia). Correo electrónico: fruizj@um.es

\section{RESUMEN}

En la creciente población de atletas veteranos españoles se analiza su ansiedad precompetitiva. Además, se evalúan qué variables de motivación, adicción, satisfacción intrínseca, percepción y creencias sobre las causas del éxito en el deporte están relacionadas con la ansiedad y la predicen. Se hallaron diferencias significativas en motivación intrínseca y ansiedad somática a favor de las mujeres y de los hombres en autoconfianza. Se discuten los resultados y se concluye que la moderada ansiedad que sufren está relacionada con la presión que quieran imponerse a sí mismos por las expectativas de marcas o puesto que pretenden lograr en competición. Esto hace que practiquen su deporte durante muchos años, pues realmente disfrutan de sus entrenamientos, esforzándose por aumentar la habilidad técnica en su especialidad atlética, lo cual les genera alta autoconfianza y mejoría del rendimiento. Por ello, con frecuencia realizan marcas en competición que hacía tiempo no lograban, incrementando sus expectativas de éxito y sensaciones de competencia, sobre todo si vencen a sus rivales. Por eso, el aburrimiento y el empleo de técnicas de engaño apenas existen en ellos. Palabras clave

autodeterminación; causas del éxito deportivo; orientación de metas; adicción; satisfacción intrínseca

\section{A B S T R A C T}

We carry out an analysis of precompetitive anxiety in the growing number of Spanish master athletes. In addition, we evaluate which variables, including motivation, addiction intrinsic satisfaction, perception and beliefs about sporting success, are related to anxiety and which can be used to predict it. Significant differences were found between women and men, the former displaying higher results in terms of intrinsic motivation and somatic anxiety, and the latter in terms of self-confidence. We discuss the results and conclude that the moderate anxiety suffered is linked to the amount of pressure that they wish to impose upon themselves, stemming from expectation of the record or place they hope to reach in a competition situation. This causes them to practice their sport for many years, and they truly enjoy their training, pushing themselves by improving their technical ability in their chosen athletic speciality, which in turn generates high self-confidence and would improve performance. For this reason, they frequently achieve records in competition which they did not reach in the past, increasing their expectations of success and sense of competence, above all if they beat their rivals. Consequently, boredom and deceptive techniques barely exist within this group.

Keywords

self-determination; causes of sport success; goal orientation; addiction; intrinsic satisfaction 


\section{Introducción}

En el deporte, la competición siempre gira en torno a dos variables: ganar o perder, conseguir o no una marca o puesto en un campeonato para lograr una medalla, un trofeo o una beca, etc. La posibilidad de no conseguir estos objetivos genera en los competidores una incertidumbre que Anshel et al. (1991) definieron como "un sentimiento subjetivo de aprensión o amenaza percibida, a veces acompañada por un incremento de la activación fisiológica" (p. 9): la ansiedad precompetitiva.

En función de cómo gestionen esta ansiedad en los momentos previos a la competición, unos deportistas bajan su rendimiento y otros lo aumentan (Smith, 1989). A estos últimos, mejores competidores, la ansiedad les parece incluso divertida, un desa fío, que les hace mejorar su rendimiento, mientras que a los primeros, peores competidores, les produce sentimientos estresantes de inseguridad o amenaza, que le hacen bajar considerablemente su rendimiento en competición (Anshel, 1995).

Para medir la ansiedad precompetitiva, se han diseñado y validado diversos instrumentos. Entre ellos destacan el Sport Competition Anxiety Test (SCAT) de Martens (1977), el Exercise Dependence Questionnaire (EDQ) de Ogden, Vaele y Summers (1997), la Exercise Dependence Scale (EDS) de Hausenblas y Downs (2002), la Sport Anxiety Scale-2 (SAS-2) de Smith, Smoll, Cumming y Grossbard (2006) y el Revised Competitive State Anxiety Inventory-2 (CSAI-2R) de Cox, Martens y Russell (2003). Este último, a diferencia de versiones previas, considera ya tres dimensiones en la ansiedad precompetitiva: ansiedad cognitiva, ansiedad somática y autoconfianza. La cognitiva, se entiende como una reacción a nivel mental causada por expectativas negativas sobre el éxito o por autoevaluación negativa en la tarea por realizar. La somática, es el conjunto de reacciones a nivel fisiológico derivados de la anterior (tensión muscular, aumento de frecuencia cardíaca, sudoración y malestar en el estómago). La tercera dimensión es la autoconfianza que, en contraposición a las dos primeras, estima el grado de seguridad que la persona cree tener acerca de sus posibilidades de éxito en la tarea que va a realizar.

Normalmente, los dos estados de ansiedad correlacionan positivamente entre sí y negativamente con la autoconfianza (Buceta, López, Pérez-Llantada, Vallejo \& Del Pino, 2003; Cervelló, SantosRosa, Jiménez, Nerea \& García, 2002) y, aunque los hombres muestran mayor autoconfianza que las mujeres, son ellas quienes controlan mejor su ansiedad (Ponce de León, López \& Medina, 2006). En la población de atletas veteranos españoles, concretamente, Zarauz y Ruiz-Juan (2013a) hallaron que hombres y mujeres presentan valores medios en ansiedad precompetitiva (cognitiva y somática) y altos en autoconfianza, encontrándose diferencias significativas a favor de las mujeres en ansiedad somática y de los hombres en autoconfianza.

En cuanto al rendimiento en competición, Burton (1988) halló una relación curvilínea de U invertida entre rendimiento y ansiedad somática, pero además una relación negativa entre ansiedad cognitiva y rendimiento, y positiva entre este y autoconfianza.

En la literatura hay multitud de estudios que relacionan la ansiedad con diversos constructos psicológicos. Así, con respecto a las motivaciones, centrándose en la Teoría de la Autodeterminación, Cecchini, González, Carmona y Contreras (2004) hallaron que la autoconfianza estaba asociada con las motivaciones intrínsecas, mientras que los dos estados de la ansiedad se asociaban con las extrínsecas. Bajo este mismo enfoque, Ruiz-Juan y Zarauz (2012a), en la población de atletas veteranos españoles, hallaron una elevada motivación intrínseca y moderada motivación extrínseca, puesto que para estos lo más importante de su práctica deportiva es la satisfacción que les produce superarse a sí mismos en los entrenamientos y, secundariamente, superar a los rivales en competición, esperando por ello una medalla y, mejor aún, un récord, todo lo cual colma sus sensaciones de competencia y expectativas de éxito.

Desde la perspectiva motivacional de metas de logro, en la ansiedad también puede influir de manera significativa la percepción del éxito, de manera que en los deportistas con alta orientación al ego 
se pueden encontrar niveles superiores de ansiedad y bajos de autoconfianza (Voigh, Callaghan \& Ryska, 2000). Por su parte, Cervelló et al. (2002) concluyeron que la orientación al ego predice significativamente de manera negativa la ansiedad cognitiva, mientras que la orientación disposicional a la tarea predecía positivamente la autoconfianza. García-Mas et al. (2011) concluyeron que existía una correlación positiva entre la orientación al ego y la ansiedad cognitiva, mientras que Olmedilla, Andreu, Ortín y Blas (2009) la obtuvieron negativa, entre la orientación a la tarea y la ansiedad rasgo.

Recientemente, Zarauz y Ruiz-Juan (2013b), en un estudio con atletas veteranos españoles, concluyeron que el perfil del veterano con mayor autoconfianza y menor ansiedad es aquel que, siendo varón de más de 50 años, muestra una mayor orientación a la tarea y diversión en la práctica de las especialidades atléticas más técnicas, y que, a su vez, se autoentrena y tiene más de 20 años de experiencia en la práctica de su prueba.

En la literatura también hay estudios que relacionan la percepción del éxito en el deporte con la satisfacción intrínseca y la percepción de las creencias sobre las causas del éxito en el deporte. Así, Castillo, Balaguer y Duda (2002) muestran que la orientación a la tarea se relaciona positivamente con la diversión y negativamente con el aburrimiento, mientras que la orientación al ego se relaciona positivamente con el aburrimiento. Por su parte, Castillo, Balaguer, Duda y García-Merita (2004) concluyen que la orientación a la tarea predice la participación deportiva a través de percepción de competencia y diversión. Posteriormente, White, Kavussanu, Tank y Wingate (2004) explican que los atletas que perciben que el esfuerzo es lo que lleva al éxito deportivo están relacionados con la orientación a la tarea, mientras que la orientación al ego está ligada a una superior habilidad, factores externos y empleo de técnicas de engaño. Además, Ruiz-Juan, Gómez, Pappous, Alacid y Flores (2010) indicaron que la orientación tarea estaba relacionada positivamente con el disfrute, y que la atribución del éxito en el deporte dependía del esfuerzo.

En el deporte de competición, además, existe otro constructo psicológico que podría estar estre- chamente relacionado con la ansiedad precompetitiva: la adicción o dependencia del entrenamiento. A esta dependencia Raedeke (1997) la llamó atrapamiento (percepción del deportista de tener que participar obligatoriamente). Por su parte, Rodríguez (2007), en la población de fisicoculturistas, la llamó vigorexia, mientras que Morgan (1977) y Ruiz-Juan y Zarauz (2012b), en el caso de los maratonianos, la llamaron, adicción negativa.

La mayoría de los investigadores coinciden en que esta dependencia, atrapamiento o adicción negativa, se debe tanto a factores de tipo psicológico (mejora del estado de ánimo, salud, autoestima, confianza y relaciones sociales) como a factores de tipo fisiológico (la activación del sistema opioide endógeno en el cerebro, la acción de las catecolaminas y la activación de estructuras cerebrales específicas), que se producen con la práctica deportiva regular (Adams \& Kirkby, 2003; Antolín, De la Gándara, García \& Martín, 2009; Arbinaga \& Caracuel, 2007; Hamer \& Karageorghis, 2007). Sin embargo, todavía no se ha llegado a conclusiones definitivas que expliquen esta adicción al deporte.

Según datos de la Real Federación Española de Atletismo ([RFEA], 2013), la población de atletas veteranos (mayores de 35 años) que entrenan y compiten habitualmente en pistas de atletismo, se ha incrementado de manera significativa en los últimos quince años. En España, sin embargo, son insuficientes los estudios sobre la ansiedad, motivaciones, adicción al entrenamiento, satisfacción, percepciones y creencias de las causas del éxito en el deporte en esta creciente población. Igualmente, se aprecian algunas divergencias en los resultados de las investigaciones internacionales sobre estos constructos psicológicos, su predicción y relaciones.

Por ello, el objetivo del presente estudio será tratar de relacionar todos estos constructos psicológicos con la ansiedad y obtener unos sólidos modelos predictivos de la misma, en función del sexo y estos mismos constructos. Para ello, se parte de la hipótesis de que en los atletas veteranos españoles existe una moderada ansiedad precompetitiva (cognitiva y somática) y alta autoconfianza. Igualmente, que se van a obtener unos sólidos modelos predictores de dichos constructos, esperando diferencias entre se- 
xos. Así, la ansiedad se podrá predecir por puntuar alto en amotivación, orientación al ego, adicción al entrenamiento, aburrimiento y engaño, así como bajo en esfuerzo. La autoconfianza por puntuar alto en motivación intrínseca, orientación a la tarea y diversión, así como bajo adicción.

\section{Método}

\section{Participantes}

Se partió del total de atletas veteranos de pista participantes en competiciones organizadas específicamente para ellos, de nivel provincial, autonómico y nacional en España en el año 2011. Según datos de la RFEA (2013), hubo 5704 licencias federativas de atletas veteranos de pista, de los cuales 4727 son hombres y 977 son mujeres.

Se obtuvo una muestra voluntaria de 401 atletas veteranos de pista, de los cuales 330 son hombres (82.3\%) con rango de edad de 35 a 75 años $(M=$ 47.98; $D E=9.14)$ y 71 mujeres $(17.7 \%)$ con rango de edad de 35 a 69 años $(M=45.78 ; D E=10.25)$. Por tanto, se tiene una muestra representativa con un error del $\pm 4.82 \%$, a un intervalo de confianza del $95.5 \%$.

\section{Procedimiento}

Para obtener una muestra lo más amplia y dispersa geográficamente en el territorio español, se pidió colaboración al responsable nacional de atletas veteranos para colgar un enlace en la sección de veteranos de la web de la RFEA (http://www.rfea.es/ veteranos/veteranos.asp), y así todos los veteranos que visitan la página puedan rellenar el cuestionario telemáticamente. Igualmente, al webmaster del principal foro de atletismo de España (http://www. elatleta.com/foro/forum.php) se le pidió que lo ubicara en la sección de atletas veteranos.

En la portada de la web (http://www.retos. org/2encuesta/inicio.html) se informaba del objetivo del estudio, de la voluntariedad, absoluta confidencialidad de las respuestas y manejo de los datos, que no había respuestas correctas o incorrectas y se les solicitó que contestaran con la máxima sinceridad y honestidad. Este trabajo posee el informe favorable de la Comisión de Bioética de la Universidad de Murcia.

\section{Instrumentos}

Inventario de Ansiedad Competitiva-2 Revisado

La versión española de Andrade, Lois y Arce (2007) del Revised Competitive State Anxiety Inventory-2 (CSAI-2R) de Cox et al. (2003) tiene 3 subescalas: ansiedad cognitiva, ansiedad somática y autoconfianza. La primera y tercera contienen cinco ítems puntuables de 1 (nada) a 4 (mucho) con puntuación total entre 5 y 20. La segunda contiene seis ítems, con puntuación total entre 6 y 24

\section{Escala de Motivación Deportiva}

Versión española de Carratalá (2003) de la Sport Motivation Scale (SMS) de Pelletier et al. (1995) que fue elaborada para medir los tres tipos de motivación intrínseca (al conocimiento, al logro y a las experiencias estimulantes), los tres tipos de motivación extrínseca (regulación externa, regulación introyectada y regulación identificada) y la amotivación. Consta de 28 ítems distribuidos en siete subescalas, correspondientes a los siete tipos de motivación mencionados, con cuatro ítems cada escala, que son respuestas a la pregunta "iPor qué practica su deporte?", puntuando en escala Likert de siete puntos desde 1 (No se corresponde en absoluto) hasta 7 (Se corresponde totalmente), con una puntuación intermedia de 4 (Se corresponde medianamente).

\section{Escala de Adicción al Entrenamiento (EAE)}

Versión adaptada al entrenamiento en el deporte por Ruiz-Juan, Zarauz y Arbinaga (2013) de la Escala de Adicción General (EAG) de Arbinaga y Caracuel (2005), consta de once ítems que valoran cuatro dimensiones relacionadas con la adicción en el deporte: placer y relajación, tolerancia, carencia de control, abstinencia y ansia. La pregunta inicial que encabeza el cuestionario es "Puntuar en una es- 
cala del 1 (muy falso) al 7 (muy cierto) las siguientes afirmaciones que mejor describan su actitud hacia el entrenamiento ...". Los resultados de la escala se agrupan en tres categorías: adicción baja (de 11 a 33 puntos), adicción media (de 34 a 55 puntos) y adicción alta (entre los 56 y los 77 puntos). La consistencia interna para las puntuaciones en el total de la escala obtenida en el estudio original fue de 0.94 .

Cuestionario de Percepción de Éxito

Versión española de Cervelló (1996) del Perception of Success Questionnaire (POSQ) de Roberts y Balagué $(1989,1991)$ que fue elaborado para medir la orientación disposicional de las metas de logro en el contexto deportivo. Consta de 12 ítems, seis de implicación disposicional en la tarea y otras seis en el ego. La pregunta inicial que encabeza el cuestionario es "Siento éxito en el deporte cuando...". Las respuestas se recogen en escala Likert que oscilan desde 1 (totalmente en desacuerdo) a 5 (totalmente de acuerdo).

\section{Cuestionario de Satisfacción \\ Intrínseca en el Deporte}

Se aplicó la versión española de Balaguer, Atienza, Castillo, Moreno y Duda (1997) del Sport Satisfaction Instrument (SSI) de Duda y Nicholls (1992). Fue elaborado para determinar el grado de divertimento. El cuestionario original consta de 8 ítems divididos en dos escalas que miden diversión ( 5 ítems) y aburrimiento (3 ítems) en la práctica deportiva. Se les pide a los sujetos que indiquen el grado de acuerdo con los ítems, recogiéndose las respuestas en escala Likert que oscila desde 1 (muy en desacuerdo) a 5 (muy de acuerdo).

Inventario de Percepción de las Creencias sobre las Causas del Éxito en el Deporte Versión española de Castillo et al. (2002) del Beliefs About the Causes of Sport Success Questionnaire (BACSSQ) de Duda y Nicholls (1992), inventario que consta de 18 ítems que miden las percepciones que tienen los sujetos sobre si el esfuerzo (9 ítems, esfuerzo ejercido en el desempeño de la tarea), la habilidad (4 ítems, factores relacionados con la posesión de habilidad) y el uso de técnicas de

\section{TABLA 1}

Coeficiente Alfa $(\alpha)$, media (M), desviación típica (DT), t, significación (p) y tamaño del efecto (d) para CSAI-2R, SMS, EAE, POSQ, SSI y BACSSQ. Diferencias por sexos (hombres $n=330$ y mujeres $n=71$ )

\begin{tabular}{|c|c|c|c|c|c|c|c|c|c|}
\hline & \multicolumn{2}{|r|}{ Total } & \multicolumn{2}{|c|}{ Hombres } & \multicolumn{2}{|c|}{ Mujeres } & & \multirow[b]{2}{*}{$p$} & \multirow[b]{2}{*}{ d } \\
\hline & $\alpha$ & $\mathrm{M} \pm \mathrm{DT}$ & $\alpha$ & $\mathrm{M} \pm \mathrm{DT}$ & $\alpha$ & $\mathrm{M} \pm \mathrm{DT}$ & & & \\
\hline CSAI-2R & & & & & & & & & \\
\hline Ansiedad cognitiva & 0.8 & $2.07 \pm 0.71$ & 0.78 & $2.05 \pm 0.70$ & 0.85 & $2.17 \pm 0.76$ & -1.30 & 0.193 & -0.16 \\
\hline Ansiedad son & 0.83 & $2.06 \pm 0.68$ & ר? & $2.02 \pm 0.67$ & 0.85 & & & & -0.31 \\
\hline $\begin{array}{l}\text { Autoconfianza } \\
\text { SMS }\end{array}$ & 0.86 & $3.10 \pm 0.65$ & 0.86 & $3.13 \pm 0.63$ & 0.87 & $2.95 \pm 0.71$ & 2.07 & 0.038 & 0.27 \\
\hline Motivación Intrínseca & 0.91 & $5.20 \pm 1.15$ & 0.91 & $5.12 \pm 1.14$ & 0.92 & $5.50 \pm 1.15$ & -2.37 & 0.018 & -0.33 \\
\hline Motiv & 0.85 & $3.88 \pm 1.04$ & 0.8 & $3.88 \pm 1$ & 0.86 & $3.88 \pm 1$. & 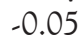 & 63 & 0 \\
\hline $\begin{array}{l}\text { Amotivación } \\
\text { EAE }\end{array}$ & 0.72 & $2.12 \pm 1.14$ & 0.71 & $2.13 \pm 1.14$ & 0.74 & $2.08 \pm 1.15$ & 0.26 & 0.792 & 0.04 \\
\hline Placer y relajación & 0.77 & $5.95 \pm 1.12$ & 0.78 & $5.95 \pm 1.13$ & 0.72 & $5.95 \pm 1.05$ & 0 & 0.993 & 0 \\
\hline & & & 0.78 & & 0.82 & & & & -0.09 \\
\hline Care & 0.77 & $5.40 \pm 1.37$ & 0.78 & $2.63 \pm 1.36$ & 0.74 & $2.50 \pm 1.40$ & 0.71 & 0.481 & 0.09 \\
\hline Abstinen & 0.71 & $3.34 \pm 1.31$ & 0.7 & $4.69 \pm 1.28$ & 0.72 & $4.52 \pm 1.44$ & 0.95 & 0.342 & 0.12 \\
\hline POS & & & & & & & & & \\
\hline Ego & 0.82 & $3.14 \pm 0.65$ & 0.81 & $3.12 \pm 0.65$ & 0.87 & $3.25 \pm 0.67$ & & & -0.20 \\
\hline $\begin{array}{l}\text { Tarea } \\
\text { SSI }\end{array}$ & 0.76 & $3.17 \pm 0.73$ & 0.76 & $3.16 \pm 0.72$ & 0.75 & $3.22 \pm 0.77$ & -0.59 & 0.557 & -0.08 \\
\hline Diversiór & 0.82 & $4.69 \pm 0.46$ & 0.84 & $4.69 \pm 0.47$ & 0.7 & 4.72 & -0.49 & 0.6 & -0.07 \\
\hline Aburr & 0.7 & $1.80 \pm 0.74$ & 0. & $1.82 \pm 0.72$ & 0.7 & $1.71 \pm 0.80$ & 1.05 & 0.294 & 0.14 \\
\hline & 0.71 & $3.44=$ & 0.71 & $3.42 \pm$ & 0.7 & 3.52 & -1. & 0.0 & -0.23 \\
\hline & & & & & & & & & 0.01 \\
\hline Engaño & 0.73 & $1.41 \pm 0.57$ & 0.7 & $1.39 \pm 0.53$ & 0.78 & $1.47 \pm 0.72$ & -0.95 & 0.342 & -0.13 \\
\hline
\end{tabular}

Fuente: elaboración propia. 
engaño (5 ítems, utilizar conductas engañosas) permiten alcanzar el éxito en el deporte. En las instrucciones se pregunta: "¿Qué crees que debería hacer la gente para tener éxito en el deporte que practica más a menudo?", debiendo responder en escala Likert desde 1 (muy en desacuerdo) a 5 (muy de acuerdo).

\section{Análisis de los datos}

Consistencia interna (alfa de Cronbach), diferencias de medias por sexo ( $t$ Student), correlaciones entre las escalas (coeficiente Pearson) y regresión lineal multivariante, se calcularon con SPSS 20.0.

\section{Resultados}

\section{Estadística descriptiva}

En la Tabla 1 se observa que los atletas veteranos españoles presentan valores medios en ansiedad precompetitiva (cognitiva y somática) y altos en autoconfianza. Igualmente, como era de esperar por el tipo de colectivo, amotivación, aburrimiento y técnicas de engaño obtienen unas puntuaciones muy bajas. Por el contrario, las puntuaciones en motivación intrínseca, diversión, placer y relajación y carencia de control son muy altas, y las obtenidas en tolerancia, esfuerzo y habilidad altas. Por último, son medias las puntuaciones obtenidas en la percepción de éxito en el deporte por ego y tarea, motivación extrínseca y abstinencia y ansia. Es reseñable que solo se han hallado diferencias significativas a favor de las mujeres en motivación intrínseca y ansiedad somática, y a favor de los hombres, en autoconfianza.

\section{Relaciones de ansiedad con motivación, adicción, percepción del éxito, satisfacción intrínseca y creencias sobre las causas del éxito en el deporte}

La Tabla 2 refleja las correlaciones calculadas. En ansiedad cognitiva y ansiedad somática se obtu-

\section{TABLA 2}

Correlaciones entre las subescalas CSAI-2R, SMS, EAE, POSQ, SSI y BACSSQ, según sexo.

\begin{tabular}{lcccccc}
\hline & \multicolumn{5}{c}{ Hombres } & \multicolumn{3}{c}{ Mujeres } \\
\cline { 2 - 7 } & $\begin{array}{c}\text { Ansiedad } \\
\text { cognitiva }\end{array}$ & $\begin{array}{c}\text { Ansiedad } \\
\text { somática }\end{array}$ & Autoconfianza & $\begin{array}{c}\text { Ansiedad } \\
\text { cognitiva }\end{array}$ & $\begin{array}{c}\text { Ansiedad } \\
\text { somática }\end{array}$ & Autoconfianza \\
\hline SMS & 0.04 & 0.05 & $0.17^{* *}$ & -0.09 & -0.08 & $0.31^{* *}$ \\
Motivación intrínseca & $0.32^{* *}$ & $0.17^{* *}$ & 0.02 & 0.18 & 0.22 & 0.16 \\
Motivación extrínseca & $0.32^{* *}$ & $0.21^{* *}$ & $-0.19^{* *}$ & $0.31^{* *}$ & $0.41^{* *}$ & -0.18 \\
Amotivación & & & & & & \\
EAE & 0 & 0.02 & 0.07 & 0.11 & 0.06 & 0.15 \\
Placer y relajación & $0.39^{* *}$ & $0.32^{* *}$ & $-0.17^{* *}$ & 0.19 & 0.16 & 0.01 \\
Tolerancia & $0.43^{* *}$ & $0.34^{* *}$ & $-0.17^{* *}$ & $0.28^{*}$ & 0.20 & 0.06 \\
Carencia de control & $0.23^{* *}$ & $0.18^{* *}$ & $-0.11^{*}$ & 0.22 & 0.03 & -0.13 \\
Abstinencia y ansia & & & & & & \\
POSQ & $0.26^{* *}$ & $0.18^{* *}$ & 0.08 & 0.17 & 0.11 & 0.20 \\
Ego & $0.15^{* *}$ & 0.08 & $0.14^{* *}$ & 0.05 & 0.1 & 0.18 \\
Tarea & & & & & & \\
SSI & -0.1 & -0.02 & $0.20^{* *}$ & 0 & -0.15 & $0.26^{*}$ \\
Diversión & $0.2^{* *}$ & $0.27^{* *}$ & $-0.14^{* *}$ & $0.24^{*}$ & $0.25^{*}$ & 0.07 \\
Aburrimiento & & & & & & \\
BACSSQ & -0.12 & 0.05 & 0.10 & 0.16 & 0.18 & 0.09 \\
Esfuerzo & $0.15^{* *}$ & $0.13^{*}$ & 0.02 & 0.22 & -0.01 & 0.13 \\
Habilidad & $0.26^{* *}$ & $0.2^{* *}$ & -0.09 & $0.32^{* *}$ & $0.38^{* *}$ & -0.02 \\
Engaño & & & & &
\end{tabular}

$*(\mathrm{p}<0.05), * *(\mathrm{p}<0.01)$

Fuente: elaboración propia. 
vieron en hombres correlaciones con casi todas las demás variables, con la excepción de motivación intrínseca, placer y relajación, diversión y esfuerzo. En mujeres, sin embargo, tanto ansiedad cognitiva como somática, únicamente correlacionaron con amotivación, aburrimiento y empleo de técnicas de engaño, así como con carencia de control sólo la ansiedad cognitiva.

En autoconfianza se halló, en hombres, correlación negativa y significativa con amotivación, tolerancia, carencia de control, abstinencia y ansia, aburrimiento y empleo de técnicas de engaño, así como positiva con motivación intrínseca, orientación a la tarea y diversión. En mujeres, sin embargo, la amotivación sólo correlacionó de manera positiva y significativa con motivación intrínseca y diversión.

\section{Análisis regresivo multivariante}

Se realizó análisis de regresión lineal multivariado, tratando de obtener unos modelos que explicasen la mayor parte posible de varianza. Se tomaron como variables dependientes la puntuación media de cada una de las subescalas del CSAI-2R (ansiedad cognitiva, ansiedad somática y autoconfianza) y como variable predictora cada una de las subescalas de motivación (intrínseca, extrínseca y amotivación), adicción al entrenamiento (placer y relajación, tolerancia, carencia de control y abstinencia y ansia), percepción de éxito (orientación a la tarea y orientación al ego), satisfacción intrínseca (diversión y aburrimiento) y percepción de las creencias sobre las causas del éxito en deporte (esfuerzo, habilidad y técnicas de engaño). Como variable de selección, se consideró el sexo.

Se extrajo el valor $R^{2}$ para explicar la varianza, Beta para explicar la predicción entre variables, $F$ para ver si existe relación entre variables seleccionadas y su significatividad (Tabla 3). Se obtienen sólidos modelos, ya que explican alrededor de la mitad de la varianza en hombres y mujeres.

El modelo muestra que ansiedad cognitiva, en hombres, se puede predecir significativamente por

\section{TABLA 3}

Análisis Regresivo Lineal Multivariado: modelos que predicen significativamente la ansiedad precompetitiva (CSAI-2R), por sexos, en función de percepción de SMS, EAE, POSQ, SSI y BACSSQ.

\begin{tabular}{|c|c|c|c|c|c|c|}
\hline & \multicolumn{2}{|c|}{ Ansiedad cognitiva } & \multicolumn{2}{|c|}{ Ansiedad somática } & \multicolumn{2}{|c|}{ Autoconfianza } \\
\hline & Hombres & Mujeres & Hombres & Mujeres & Hombres & Mujeres \\
\hline & BetaSign & BetaSign & BetaSign & BetaSign & BetaSign & BetaSign \\
\hline \multicolumn{7}{|l|}{ SMS } \\
\hline Motivación Intrínseca & 0.05 & -0.13 & 0.03 & -0.01 & 0.10 & $0.31 * * *$ \\
\hline Motivación Extrínseca & 0.07 & 0 & -0.01 & 0.14 & 0.03 & 0 \\
\hline \multicolumn{6}{|l|}{ EAE } & -0.13 \\
\hline Placer y relajación & -0.02 & 0.01 & -0.02 & 0.12 & 0.02 & 0.02 \\
\hline Tolerancia & $0.22 * * *$ & 0.02 & $0.16 * *$ & 0.08 & -0.10 & 0.01 \\
\hline Carencia de control & $0.22 * * *$ & 0.08 & $0.20 * *$ & 0.08 & $-0.20 * * *$ & 0.06 \\
\hline Abstinencia y ansia & 0.09 & $0.24 *$ & 0.03 & 0.05 & -0.09 & -0.12 \\
\hline \multicolumn{7}{|l|}{ POSQ } \\
\hline Ego & $0.16 * *$ & 0.09 & 0.06 & 0.07 & 0.04 & 0.11 \\
\hline Tarea SSI & 0.02 & -0.01 & 0.04 & 0.07 & $0.16 * *$ & 0.09 \\
\hline Diversión & -0.01 & 0.01 & 0.01 & 0 & $0.20 * * *$ & 0.14 \\
\hline Aburrimiento & 0.01 & 0.05 & $0.17 * *$ & 0.13 & -0.07 & 0.04 \\
\hline \multicolumn{7}{|l|}{ BACSSQ } \\
\hline Esfuerzo & $-0.16 * *$ & 0.03 & -0.02 & 0.05 & 0.05 & -0.04 \\
\hline Habilidad & 0 & 0.08 & 0.05 & -0.10 & -0.01 & 0.10 \\
\hline \multirow{3}{*}{ Engaño } & $0.16^{*}$ & $0.34 * *$ & $0.11^{*}$ & 0.24 & -0.02 & -0.08 \\
\hline & $\mathrm{R} 2=0.558$ & $\mathrm{R} 2=0.407$ & $\mathrm{R} 2=0.436$ & $\mathrm{R} 2=0.406$ & $\mathrm{R} 2=0.313$ & $\mathrm{R} 2=0.307$ \\
\hline & $F=24.376$ & $\mathrm{~F}=6.748$ & $\mathrm{~F}=19.041$ & $F=13.652$ & $\mathrm{~F}=11.778$ & $\mathrm{~F}=7.182$ \\
\hline
\end{tabular}

$*(\mathrm{p}<0.05), * *(\mathrm{p}<0.01), * * *(\mathrm{p}<0.001)$

Fuente: elaboración propia. 
puntuar alto en amotivación, tolerancia, carencia de control, orientación al ego y bajo, en esfuerzo (55.8\% varianza). Sin embargo, en mujeres la predicción fue únicamente por puntuar alto en abstinencia y ansia, así como en empleo de técnicas de engaño (40.7\% de varianza).

El modelo de ansiedad somática, en hombres, muestra que se puede predecir significativamente por puntuar alto en tolerancia, carencia de control, aburrimiento y empleo de técnicas de engaño $(43.6 \%$ varianza), mientras que en mujeres lo es únicamente por puntuar alto en amotivación (40.6\% varianza).

El modelo de autoconfianza, en hombres, indica que se puede predecir significativamente por puntuar bajo en carencia de control y alto en diversión (31.3\% de varianza). En mujeres, sin embargo, lo es sólo por puntuar bajo en motivación intrínseca (30.7\% de varianza).

\section{Discusión}

Por sus hábitos de entrenamiento, los veteranos españoles son unos deportistas que se toman muy en serio su actividad atlética (Ruiz-Juan \& Zarauz, 2012a; Zarauz \& Ruiz-Juan, 2013a), formando esta una parte muy importante dentro de su vida. Por ello, como se predijo, al igual que obtuvo Leedy (2000) en corredores muy comprometidos, esto hace que tengan una moderada ansiedad, tanto cognitiva como somática. Igualmente, que tengan una elevada autoconfianza, sobre todo los hombres, como obtuvieron Ponce de León et al. (2006).

Las altas puntuaciones en diversión, esfuerzo y habilidad, las moderadas en percepción del éxito y las bajas en aburrimiento y uso de técnicas de engaño pueden quedar explicadas por el hecho de que estos atletas tienen una elevada motivación intrínseca y moderada motivación extrínseca para entrenar y competir (Ruiz-Juan \& Zarauz, 2012a). El equilibrio obtenido en las puntuaciones de la percepción del éxito explica que para los atletas veteranos es tan importante la satisfacción que les produce superarse a sí mismos en entrenamientos como superar a rivales en competición, esperando por ello una medalla o un récord. Todo ello incre- menta sus sensaciones de competencia y expectativas de éxito.

Contrariamente a los atletas profesionales, al no depender los veteranos de motivaciones extrínsecas (becas o contratos publicitarios por lograr grandes marcas), la inseguridad, estrés, aprensión o amenaza (ansiedad cognitiva) o la tensión muscular, aumento de frecuencia cardíaca, sudoración y malestar en el estómago (ansiedad somática) que perciben ante la proximidad del momento de competir, será menor que la de los atletas profesionales (Smith, 1989). Si a ello le sumamos una mayor edad y experiencia, inevitablemente serán "mejores competidores" que los atletas noveles, como señalaba Anshel (1995). Esto explica que en los atletas veteranos aumente significativamente la diversión, esfuerzo y habilidad y disminuya el aburrimiento y empleo de técnicas de engaño.

Las correlaciones obtenidas entre la ansiedad y el resto de variables se han producido dentro de lo esperado, puesto que normalmente la ansiedad se relaciona con motivaciones extrínsecas y/o amotivación y la autoconfianza con las motivaciones más autodeterminadas, en la línea con lo obtenido por Cecchini et al. (2004).

Se destaca que en hombres se ha producido una lógica relación positiva entre las tres subescalas de adicción al entrenamiento menos deseables (tolerancia, carencia de control y abstinencia y ansia) con los dos estados de ansiedad (cognitiva y somática), pero no así en mujeres (salvo por la relación positiva en ellas de la carencia de control con la ansiedad cognitiva). Por tanto, se confirman las claras diferencias por género obtenidas en la mayoría de las investigaciones (Ponce de León et al., 2006; Zarauz \& Ruiz-Juan, 2013a) y con las que se contaba en la hipótesis.

Igualmente, es reseñable en ambos sexos, la significativa relación positiva entre los dos estados de ansiedad con el aburrimiento y el empleo de técnicas de engaño y, solo en hombres, con la percepción de éxito por orientación al ego y la demostración de una superior habilidad, al igual que Voigh et al. (2000) y White et al. (2004), respectivamente. Ello puede quedar explicado porque los atletas con alta orientación al ego no consideran relevantes la 
diversión y el esfuerzo en el desempeño de su actividad deportiva, según Cervelló, Escartí y Balagué (1999). Más bien, perciben que la causa del éxito es demostrar superior habilidad, justificándose incluso el empleo de técnicas de engaño para conseguirlo. Por eso, los atletas veteranos españoles con mayor orientación al ego desarrollan mayor ansiedad precompetitiva.

También es importante destacar que, en ambos sexos, la autoconfianza correlaciona positivamente con la diversión y, solo en hombres, con la percepción de éxito por orientación a la tarea, como obtuvieron Castillo et al. (2002), Castillo et al. (2004) y Ruiz-Juan et al. (2010). Esto podría deberse a que los deportistas con alta orientación a la tarea, consideran el esfuerzo (y no el engaño) la principal causa del éxito en el deporte, sintiéndose muy satisfechos simplemente con mejorar su habilidad deportiva (Cervelló et al., 1999). Por ello, de manera similar a lo obtenido por Cervelló et al. (2002), puede afirmarse que los atletas veteranos con mayor orientación a la tarea tienen mayor autoconfianza y, por consiguiente, mayor rendimiento y probabilidad de conseguir sus objetivos, como concluía Smith (1989).

En cuanto a los resultados obtenidos en los modelos predictivos de ansiedad y autoconfianza, vale mencionar que coinciden en gran medida con lo planteado en la hipótesis y con los resultados de Castillo et al. (2004), Cervelló et al. (2002), RuizJuan et al. (2010), Voigh et al. (2000), White et al. (2004) y Zarauz y Ruiz-Juan (2013b). Así, de estos modelos predictivos se puede extraer que, cuando puntualmente estos atletas perciben que no han alcanzado un estado de forma o habilidad óptimas para un campeonato, disminuye su motivación y se les produce la moderada ansiedad precompetitiva que sufren, sobre todo cuando va unida a alta orientación ego. Además, muestran que al atleta veterano español le divierte la práctica de su especialidad atlética, ya sea en entrenamiento o en competición, esforzándose por incrementar su habilidad día a día, lo cual le genera alta autoconfianza y sensación de competencia que, como explicaba Burton (1988), le ayuda a mejorar su rendimiento. Todo esto puede ser explicado porque su motivación es significativamente autodeterminada (Ruiz-Juan $\&$ Zarauz, 2012a), lo cual produce que esta población prolongue en el tiempo su actividad deportiva.

A modo de conclusiones, se indica que, como estos atletas no dependen de motivaciones externas de tipo económico (se autofinancian los gastos derivados de su actividad deportiva), la moderada ansiedad precompetitiva que sufren está relacionada con la presión que quieran imponerse a sí mismos por las expectativas de marcas o puesto que pretenden lograr en competición. Esto hace que practiquen su deporte durante muchos años, pues realmente disfrutan de sus entrenamientos, esforzándose por aumentar la habilidad técnica en su especialidad atlética, lo cual les genera alta autoconfianza y mejoría del rendimiento. Por ello, con frecuencia realizan marcas en competición que hacía tiempo no lograban, incrementando sus expectativas de éxito y sensaciones de competencia, sobre todo si vencen a sus rivales, aunque para ellos no es lo más importante. Por eso, el aburrimiento y el empleo de técnicas de engaño apenas existen en esta población.

Para investigaciones futuras queda un vasto campo de investigación abierto para estudiar la ansiedad y autoconfianza de los atletas veteranos españoles con otras variables comparativas de interés: percepción del estado físico, hábitos de entrenamiento, expectativas de éxito, algunas variables sociodemográficas, etc., dada la insuficiente bibliografía existente en esta creciente población.

\section{Referencias}

Adams, J., \& Kirkby, R. J. (2003). El exceso de ejercicio como adicción: una revisión. Revista de Toxicomanías, 34, 10-23.

Andrade, E. M., Lois, G., \& Arce, C. (2007). Propiedades psicométricas de la versión española del Inventario de Ansiedad Competitiva CSAI-2R en deportistas. Psicothema, 19(1), 150-155.

Anshel, M. (1995). Anxiety. En T. Morris \& J. Summers (Eds.), Sport psychology: Theory, applications $\mathcal{E}$ issues (pp. 29-62). Brisbane, CA: John Wiley \& Sons.

Anshel, M., Freedson, P., Hamill, J., Haywood, K., Horvat, M., \& Plowman, S. (1991). Dictionary 
of the sport and exercise sciences. Champaign, IL: Human Kinetics.

Antolín, V., De la Gándara, J. J., García, I., \& Martín, A. (2009). Adicción al deporte: imoda postmoderna o problema sociosanitario? Norte de Salud Mental, 1(34), 15-22.

Arbinaga, F., \& Caracuel, J. C. (2005). Precompetición y ansiedad en fisicoculturistas. Revista de Psicología del Deporte, 14(2), 195-208.

Arbinaga, F., \& Caracuel, J. C. (2007). Dependencia del ejercicio en fisicoculturistas competidores evaluada mediante la escala de adicción Ramón y Cajal. Universitas Psychologica, 6(3), 549-558.

Balaguer, I., Atienza, F. L., Castillo, I., Moreno, Y., \& Duda, J. L. (1997, septiembre). Factorial structure of measures of satisfaction/interest in sport and classroom in the case of Spanish adolescents. Trabajo presentado en la Fourth European Conference of Psychological Assessment, Lisboa, Portugal.

Buceta, J., López, A., Pérez-Llantada, M., Vallejo, M., \& Del Pino, M. (2003). Estado psicológico de los corredores populares de maratón en los días anteriores a la prueba. Psicothema, 15(2), 273-277.

Burton, D. (1988). Do anxious swimmers swim slower? Reexamining the elusive anxiety-performance relationship. Journal of Sport and Exercise Psychology, 10, 45-61.

Carratalá, E. (2003). Análisis de la teoría de las Metas de Logro y de la Autodeterminación en los planes de especialización deportiva de la Generalitat Valenciana. Tesis Doctoral sin publicar, Facultad de Psicología, Universidad de Valencia, Valencia, España.

Castillo, I., Balaguer. I., \& Duda, J. (2002). Las perspectivas de meta de los adolescentes en el contexto deportivo. Psicothema, 14(2), 280-287.

Castillo, I., Balaguer. I., Duda, J., \& García-Merita, M. (2004). Factores psicosociales asociados con la participación deportiva en la adolescencia. Revista Latinoamericana de Psicología, 36(3), 505-515.

Cecchini, J. A., González, C., Carmona, A. M., \& Contreras, O. (2004). Relaciones entre clima motivacional, la orientación de meta, la motivación intrínseca, la auto-confianza, la ansiedad y el estado de ánimo en jóvenes deportistas. Psicothema, 16(1), 104-109.
Cervelló, E. (1996). La motivación y el abandono deportivo desde la perspectiva de las metas de logro. Tesis Doctoral, Universidad de Valencia, Valencia, España.

Cervelló, E., Escartí, A., \& Balagué, G. (1999). Relaciones entre la orientación de meta disposicional y la satisfacción con los resultados deportivos, las creencias sobre las causas de éxito en deporte y la diversión con la práctica deportiva. Revista de Psicología del Deporte, 8(1), 7-19.

Cervelló, E., Santos-Rosa, F. J., Jiménez, R., Nerea, A., \& García, T. (2002). Motivación y ansiedad en jugadores de tenis. Motricidad, 9, 141-161.

Cox, R. H., Martens, M. P., \& Russell, W. D. (2003). Measuring anxiety in athletics: The revised Competitive State Anxiety Inventory-2. Journal of Sport and Exercise Psychology, 25(4), 519-533.

Duda, J. L., \& Nicholls, J. G. (1992). Dimensions of achievement motivation in schoolwork and sport. Journal of Educational Psychology, 84(3), 290-299.

García-Mas, A., Palou, P., Smith, R. E., Ponseti, X., Almeida, P., Lameiras, J., Jiménez, R., et al. (2011). Ansiedad competitiva y clima motivacional en jóvenes futbolistas de competición, en relación con las habilidades y el rendimiento percibido por sus entrenadores. Revista de Psicología del Deporte, 20(1), 197-207.

Hamer, M., \& Karageorghis, C. (2007). Psychobiological mechanisms of exercise dependence. Sports medicine, 37(6), 477-484.

Hausenblas, H. A., \& Downs, D. S. (2002). How much is too much? The development and validation of the Exercise Dependence Scale. Psychology \& Health, 17(4), 387-404.

Leedy, M. (2000). Commitment to distance running: Coping mechanism or addiction? Journal of Sport Behavior, 23(3), 255-270.

Martens, R. (1977). Sport Competition Anxiety Test. Chanpaing, IL: Human Kinetics.

Morgan, W. P. (1979). Negative addiction in runners. The Physician and Sports Medicine, 7, 57-77.

Ogden, J., Veale, D., \& Summers, Z. (1997). The development and validation of the exercise dependence questionnaire. Addiction Research, 5(4), 343-356.

Olmedilla, A., Andreu, M. D., Ortín, F. J., \& Blas, A. (2009). Ansiedad competitiva, percepción de éxito y lesiones: un estudio en futbolistas. Revista 
Internacional de Medicina y Ciencias de la Actividad Física y el Deporte, 9(33), 51-66.

Pelletier, L. G., Fortier, M. S., Vallerand, R. J., Tuson, K. M., Brière, N. M., \& Blais, M. R. (1995). Toward a new measure of intrinsic motivation, extrinsic motivation, and amotivation in sports: The Sport Motivation Scale (SMS). Journal of Sport and Exercise Psychology, 17, 35-53.

Ponce de León, Y., López, J. \& Medina, M. (2006). Habilidades psicológicas en los atletas de primera fuerza en atletismo. Revista de Ciencias del Ejercicio, 2(1), 42-57.

Raedeke, T. D. (1997). Is athlete burnout more than just stress? A sport commitment perspective. Journal of Sport EJ Exercise Psychology, 19(4), 396-417.

Real Federación Española de Atletismo. (2013). Comparativa histórica de licencias de veteranos. Recuperado de http://www.rfea.es/veteranos/licencias.asp

Roberts, G. C., \& Balagué, G. (1989, agosto). The development of a social-cognitive scale in motivation. Trabajo presentado en el Seventh World Sport Psychology Congress, Singapore.

Roberts, G. C., \& Balagué, G. (1991, septiembre). The development and validation of the Perception of Success Questionnaire. Trabajo presentado en el VIII European Federation of Sport Psychology Congress, Colonia, Alemania.

Rodríguez, J. M. (2007). Vigorexia: adicción, obsesión o dismorfia: un intento de aproximación. Salud y Drogas, 7(2), 289-308.

Ruiz-Juan, F., Gómez, M., Pappous, A., Alacid, F., \& Flores, G. (2010). Dispositional goal orientation, beliefs about the causes of success and intrinsic satisfaction in young elite players. Journal of $\mathrm{Hu}$ man Kinetics, 26, 122-136.
Ruiz-Juan, F., \& Zarauz, A. (2012a). Predictors variables of motivation in the Spanish master athletes. Journal of Human Sport and Exercise, 7(3), 617-628.

Ruiz-Juan, F., \& Zarauz, A. (2012b). Variables que hacen adicto negativamente a correr al maratoniano español. Retos, 21, 38-42.

Ruiz-Juan, F., Zarauz, A., \& Arbinaga, F. (2013). Validación de la Escala de Adicción al Deporte en atletas veteranos. Adicciones, 25(4), 309-320.

Smith, R. (1989). Athletic stress and burnout: Conceptual models and intervention stra tegies. En D. Hackfort \& C. D. Spielberger (Eds.), Anxiety in sports: An international perspective (pp. 183-201). Nueva York: Hemisphere.

Smith, R. E., Smoll, F. L., Cumming, S. P., \& Grossbard, J. R. (2006). Measurement of multidimensional sport performance anxiety in children and adults: The Sport Anxiety Scale-2. Journal of Sport and Exercise Psychology, 28(4), 479-501.

Voight, M. R., Callaghan, J. L., \& Ryska, T.A. (2000). Relationship between goal orientations, self-confidence and multidimensional trait anxiety among Mexican-American female youth athletes. Journal of Sport Behavior, 23(3), 271-288.

White, S., Kavussanu, M., Tank, K., \& Wingate, J. (2004). Perceived parental beliefs about the causes of success in sport: Relationship to athletes' achievement goals and personal beliefs. Scandinavian Journal of Medicine $\mathbb{E}$ Sciences in Sports, 14(1), 57-66.

Zarauz, A., \& Ruiz-Juan, F. (2013a). Variables predictoras de la ansiedad en atletas veteranos españoles. Retos, 23, 29-32.

Zarauz, A., \& Ruiz-Juan, F. (2013b). Análisis de la ansiedad en el atletismo: un estudio con veteranos. Revista Internacional de Ciencias del Deporte, 9(33), 222-235. 
